\begin{tabular}{|c|l|}
\hline Title & Dynamical aspect of entropy transfer in free convection turbulence \\
\hline Author(s) & Toh, Saday oshi; lima, Makoto \\
\hline Citation & $\begin{array}{l}\text { Physical Review E, 61(3), 2626-2639 } \\
\text { https://doi.org/L0.1103/PhysRevE.61.2626 }\end{array}$ \\
\hline Issue Date & 2000-03 \\
\hline Doc URL & http://hdl.handle.net/2115/44434 \\
\hline Type & article \\
\hline File Information & PhysRevE.61.2626.pdf \\
\hline
\end{tabular}

Instructions for use 


\title{
Dynamical aspect of entropy transfer in free convection turbulence
}

\author{
Sadayoshi Toh \\ Division of Physics and Planetary Sciences, Graduate School of Science, Kyoto University, Kyoto 606-8502, Japan \\ Makoto Iima \\ Research Institute for Electronic Science, Hokkaido University, Kita 12 Nishi 6, Kita-ku, Sapporo 060-0812, Japan
}

(Received 5 August 1999)

\begin{abstract}
From a dynamical perspective, entropy transfer processes are investigated in two-dimensional free convection turbulence in comparison with an entropy cascade picture based on the coupled dynamics of the $T$ vorticity $\boldsymbol{\chi} \equiv\left(\partial_{y} T,-\partial_{x} T\right)$ and the velocity gradient tensor. Typical entropy transfer processes are observed in direct numerical simulations. For these processes, two characteristic times, the transfer time and the staying time are determined: the former time obeys a Bolgiano-Obukhov (BO) time scaling corresponding to the eddy turnover time in energy cascade. It is suggested that this typical transfer process is not an elementary process of cascade but a dynamical manifestation of intermittency. To examine the meaning of the characteristic times of the typical entropy transfer process, a shell model is constructed based on the entropy cascade picture. By this model, it is shown numerically that typical entropy transfer processes are regarded as the fluctuations satisfying a dynamical similarity. This similarity proved by perturbation analysis requires naturally that the transfer time should obey the BO time scaling.
\end{abstract}

PACS number(s): 47.27.Ak, 47.27.Gs, 47.27.Eq, 47.27.Te

\section{INTRODUCTION}

Cascade is a well-known idea of turbulence. Though this idea is based on statistics and scaling, cascade is explained schematically as the successive breakdown of a mother eddy into daughter eddies within a so-called eddy turnover time: Richardson's picture [1]. This schematic picture of cascade contains a kind of dynamics, i.e., the existence of an elementary process of cascade is implicitly assumed. The cascade process is not spatially homogeneous even in a statistical sense because of intermittency, although the characteristics of the intermittency have not yet been clarified. The model proposed by She and Lévêque [2], which is believed to give an excellent explanation of anomalous scaling due to intermittency, is based on the existence of coherent structures or singular structures. In direct numerical simulations (DNS's) of the three-dimensional (3D) Navier-Stokes (NS) equations, coherent structures such as tubelike vortices are observed that play an important role especially when cascade processes are examined dynamically. However, it is not clear whether these coherent structures are directly related to the eddies or vortices mentioned in Richardson's picture, or to intermittency models. We believe research into the cascade process from the dynamical aspect should be helpful to understand fully developed turbulence. In this paper, we try to understand the meaning of the turnover time in the cascade process as a preliminary work.

In the 3D NS system, the characteristic time based on the Kolmogorov 1941 theory (K41) [3] has been considered as the "eddy turnover time" in the context of Richardson's energy cascade picture [1], in which an eddy is distorted or broken, exciting smaller eddies. In this picture, the characteristic time can be interpreted in two ways. One is the distortion time, i.e., the time for an eddy to be distorted. The other is the circulation time, i.e., the time of an eddy to exist: this is the staying time or lifetime. These two characteristic times are considered identical in the traditional cascade picture. In this situation, we define the transfer time as the time in which an appreciable part of the kinetic energy of an eddy is transferred to smaller eddies because only two generations - mother and daughter-are considered. Daughter eddies succeed to all the energy possessed by the mother before her grandchildren are born. Furthermore the localness of interactions among different scales is taken strictly into account.

We can consider another situation where many generations coexist but energy exchange is still limited among the mother and her daughters, i.e. the localness of the energy transfer still holds. Then in general the active period of an eddy is shorter than her lifetime. In this situation, the transfer time may be defined as the time taken by alternation of generations because this definition is a natural extension of the above case. Thus the transfer time is shorter than the staying time. If a blob of energy possessed by a large eddy starts to transfer to small eddies, we will observe a successive excitation of new generations of smaller eddies in the inertial range. This situation is a little bit curious, since relatively strong correlation among many generations is maintained during the transfer process even under the restriction of the localness of interactions. However, there is no reason to disregard it. In fact, we will report observations which seem to correspond to this situation. Of course, it is still an open question whether elementary processes constituting cascade exist in the dynamical sense.

In this paper we deal with $2 \mathrm{D}$ free convection (FC) turbulence instead of 3D NS turbulence for simplicity. FC is a model of the central region of hard turbulence (HT) [4-7] which is proposed to examine the power spectrum of temperature fluctuations, $P(\omega) \sim \omega^{-1.4}$. This power spectrum is explained by Bolgiano-Obukhov (BO) scaling $[8,9]$ based on the entropy $\left(T^{2} / 2\right)$ cascade [10-12]. The governing equa- 
tions of the FC system are based on the Boussinesq approximations

$$
\begin{gathered}
\frac{D \boldsymbol{u}}{D t}=-\boldsymbol{\nabla} p+\alpha g T \boldsymbol{e}_{y}+\nu \Delta \boldsymbol{u}, \\
\frac{D T}{D t}=\kappa \Delta T, \\
\boldsymbol{\nabla} \cdot \boldsymbol{u}=0,
\end{gathered}
$$

where $\boldsymbol{\nabla} \equiv(\partial / \partial x, \partial / \partial y), \Delta \equiv \boldsymbol{\nabla}^{2}$, and $D / D t \equiv \partial / \partial t+(\boldsymbol{u} \cdot \boldsymbol{\nabla})$, and $\nu, \kappa, \alpha$, and $g$ are the kinematic viscosity, the heat diffusivity, the volume expansion coefficient, and the gravitational acceleration, respectively. The buoyancy acts along the $y$ axis, and $\boldsymbol{e}_{y}$ is the unit vector of this direction. This model assumes a neutrally stable stratification and homogeneity which is realized in the central region of hard turbulence [11].

The FC system has conserved quantities, entropy $S$ $\equiv \int|T|^{2} / 2 d V$ and total energy $E \equiv \int|\boldsymbol{u}|^{2} / 2+(\alpha g) y T d V$, in the inviscid case. It should be noted that both $2 \mathrm{D}$ and $3 \mathrm{D}$ FC systems have the same conserved quantities, unlike NS systems. In the inertial range, the BO spectra for entropy and kinetic energy $S(k)$ and $E(k)$ are obtained based on entropy cascade:

$$
\begin{aligned}
& S(k) \sim \epsilon_{T}^{4 / 5}(\alpha g)^{-2 / 5} k^{-7 / 5}, \\
& E(k) \sim \epsilon_{T}^{2 / 5}(\alpha g)^{4 / 5} k^{-11 / 5},
\end{aligned}
$$

where $\epsilon_{T}$ is the average dissipation rate of the entropy.

For both 2D FC and 3D NS, phenomena related to the transfer time (the eddy turnover time) were observed. In the 2D FC system, the transfer time was estimated in terms of the correlation time using orthogonal wavelet [13]. The transfer time obtained is well explained by the the characteristic time due to $\mathrm{BO}$ scaling:

$$
\tau_{l} \sim \epsilon_{T}^{-1 / 5}(\alpha g)^{-2 / 5} l^{2 / 5} .
$$

In a 3D NS system with high symmetry, large fluctuations of the cumulative transfer over a shell in Fourier space are observed to be activated successively in scales due to the characteristic time $\tau_{l} \sim l^{2 / 3}$, which is the eddy turnover time based on K41 [14].

We expect that FC turbulence has the nature of a cascade similar to that of 3D NS turbulence. Pumir and Siggia showed that the 2D inviscid Boussinesq approximation equation corresponds to the local approximation of the Euler equation for 3D axisymmetric swirl flow to examine the existence of a finite time singularity [15]. It should be noted that the existence of the singularity is still an open question $[16,17]$, as is the relation between the singularity and the cascade.

In experiments and DNS's in 3D NS turbulence, highly vortical regions such as a vortex tube or sheet are observed and assumed structures. Their dynamics, such as stretching and tilting is believed to play an important role in energy cascade. In the 2D FC system, however, there is no picture based on structures for the entropy cascade because structures have not yet been defined. Nonetheless, the equation for $\boldsymbol{\chi} \equiv\left(\partial_{y} T,-\partial_{x} T\right)$ (called $T$ vorticity hereafter) is similar to that for vorticity in the 3D NS case like divorticity in the 2D NS system. By this equation, we will dig into the entropy cascade mechanism based on the dynamics of the structures of the $T$ vorticity.

By a shell model for FC turbulence, entropy cascade has been also examined $[18,19]$. Brandenburg concluded that backward energy transfer is crucial for reproducing the $\mathrm{BO}$ scaling in this shell model [18]. The universality of the BO spectra and the intermittency of the transfer process were also investigated [19]. Another shell model is proposed by the authors to explain the behavior of the characteristic times of the entropy transfer observed in DNS's of 2D FC turbulence. This shell model is constructed based on the evolution equations of $T$ vorticity and velocity gradient tensor [cf. Eqs. (2.1) and (2.2)]. This model is not a chaotic system, and has a stable steady solution containing the BO spectra in its inertial range.

In this paper, we examine the entropy cascade by both DNS's and the shell model. In Sec. II, we propose an entropy cascade picture based on the coupled dynamics of the $T$ vorticity and velocity gradient. We compare this picture with DNS's of the 2D FC system in Sec. III. In Sec. IV, we review our shell model and then show the results of a simulation. Section V is devoted to examining the self-similar nature of time evolution in the shell model by perturbation theory. In Sec. VI, we discuss the entropy cascade based on the two types of the entropy transfer observed in our shell model.

\section{AN ENTROPY CASCADE PICTURE}

The DNS of the 2D FC equations [Eqs. (1.1) and (1.2)] shows that the BO scaling can be realized: the entropy cascade can exist. If the cascade is governed by a universal mechanism and in this sense the entropy cascade is similar to the energy cascade, there may be physical quantities, like vorticity in the NS system, by which the entropy cascade mechanism will be understood more easily and deeply from the dynamical aspect. Then we focus on the similarity between the vorticity equation in the 3D NS system and the equation for $T$ vorticity in the 2D FC system.

To study the FC system in terms of the $T$ vorticity and velocity gradient tensor, we rewrite Eqs. (1.1) and (1.2) with $\boldsymbol{\chi}$ and $\boldsymbol{\sigma} \equiv \nabla^{t}: \boldsymbol{u}$ as follows:

$$
\begin{gathered}
\frac{D \boldsymbol{\sigma}}{D t}=\alpha g R\left(\frac{\pi}{2}\right):\left(\boldsymbol{\chi}: \boldsymbol{e}_{y}^{t}\right)-\boldsymbol{\sigma}: \boldsymbol{\sigma}-\boldsymbol{\nabla}: \boldsymbol{\nabla}^{t} p+\nu \Delta \boldsymbol{\sigma}, \\
\frac{D \boldsymbol{\chi}}{D t}=\boldsymbol{\chi} \cdot \boldsymbol{\nabla} \boldsymbol{u}+\kappa \Delta \boldsymbol{\chi}(=\boldsymbol{\sigma}: \boldsymbol{\chi}+\kappa \Delta \boldsymbol{\chi}),
\end{gathered}
$$

where $\boldsymbol{\sigma}$ is the velocity gradient tensor $\left([\boldsymbol{\sigma}]_{i j}=\partial_{i} u_{j}\right), t$ indicates a transpose, and $R(\theta)$ is the rotation matrix through angle $\theta$. The symbol : denotes the tensor product. Equation (2.2) shows that $\boldsymbol{\chi}$ is expected to play the same role as vorticity in the 3D NS system. The divorticity in the 2D NS system is also governed by Eq. (2.2), but divorticity is directly linked to velocity unlike in the $2 \mathrm{D}$ FC case. This difference in the degree of freedom between the 2D NS and 2D FC systems seems to be crucial for the cascade. 
We should illustrate the fact that it is inevitable to explain our entropy cascade picture. In the 2D FC system, potential energy is always converted into kinetic energy via the buoyancy effect in a statistical sense. Furthermore kinetic energy converted is inversely transferred to large scales. Thus the buoyancy term dominates the nonlinear term on the righthand side of Eq. (2.1). If a scale is given, the induction time of the velocity gradient tensor due to the $T$ vorticity is longer than the excitation time of the $T$ vorticity of smaller scale by the term $\boldsymbol{\sigma}: \boldsymbol{\chi}$. This fact is shown in Sec. III.

We can easily draw a picture of the entropy cascade in accordance with the form of Eqs. (2.1) and (2.2). Suppose there is a $T$ vorticity fluctuation of a certain size. Then a velocity gradient fluctuation of about the same size is excited due to the linear term in Eq. (2.1), i.e., the buoyancy. While the velocity gradient is induced, another $T$ vorticity fluctuation of a smaller size is excited by the nonlinear interaction due to $\boldsymbol{\sigma}: \boldsymbol{\chi}$. The size of the excited fluctuation of the $T$ vorticity is about half. In this picture, the interactions are closed locally in scale and the entropy is transferred to smaller scales scale by scale, like the successive breakdown of eddies shown in Richardson's cascade picture. Therefore we expect that a model based on the equations of the $T$ vorticity and velocity gradient is suitable for understanding the entropy cascade. Moreover, we believe that the explanation of the cascade process by such an entropy cascade picture will conversely be helpful in understanding energy cascade.

Here, we do not refer to a nonlinear term $-\boldsymbol{\sigma}: \boldsymbol{\sigma}$, because the buoyancy terms dominate the nonlinear term. Advection terms for each $\boldsymbol{\chi}$ and $\boldsymbol{\sigma}$ are neglected by regarding that they cause mainly a local sweeping effect. We also neglected pressure in this picture for simplicity, although the nonlocal effect of pressure might be important. The results of DNS's, however, show that pressure is not so effective in local transfer of entropy. These results will be reported elsewhere.

\section{CHARACTERISTIC TIMES IN 2D FC}

In this section, we analyze the entropy transfer process in FC turbulence from the perspective of the dynamics of the $T$ vorticity $\boldsymbol{\chi}$ and velocity gradient tensor $\boldsymbol{\sigma}$ defined in Sec. I. In particular, we focus on characteristic times related to the entropy transfer process. To describe temporal evolutions of the $T$ vorticity and velocity gradient tensor in scales, we introduce the scalar representations of these quantities as simply as possible.

\section{A. Representations of the $T$ vorticity and velocity gradient tensor}

We introduce the representations of the $T$ vorticity and velocity gradient tensor of a scale in terms of a $2 \mathrm{D}$ wavelet (cf. Appendix A for a 2D wavelet transform). However, we do not examine the details of the spatial distributions in this paper. The representations of the $T$ vorticity and velocity gradient tensor of a scale $l_{m} \sim 2^{-m}$ are referred to as $X_{m}$ and $\Sigma_{m}$, respectively. In Sec. IV, we construct a shell model for scalar variables $\sigma_{m}$ and $\chi_{m}$ corresponding to $\Sigma_{m}$ and $X_{m}$.

First of all, we define the modal wavelet velocity $\boldsymbol{u}_{m, j}(x, y)$ and the modal wavelet temperature $\Theta_{m, j}(x, y)$ in physical space as

$$
\begin{aligned}
& \boldsymbol{u}_{m, j}(x, y)= {\left[u_{m, j}(x, y), v_{m, j}(x, y)\right] } \\
& \equiv\left(\sum_{q=1}^{3} \hat{u}_{m, j}^{(q)} \Psi_{m, j}^{(q)}(x, y), \sum_{q=1}^{3} \hat{v}_{m, j}^{(q)} \Psi_{m, j}^{(q)}(x, y)\right), \\
& \\
& \Theta_{m, j}(x, y) \equiv \sum_{q=1}^{3} \hat{\Theta}_{m, j}^{(q)} \Psi_{m, j}^{(q)}(x, y),
\end{aligned}
$$

where $\hat{u}_{m, j}^{(q)}, \hat{v}_{m, j}^{(q)}$, and $\hat{\Theta}_{m, j}^{(q)}$ are wavelet coefficients of $u, v$, and $T$, respectively. Then the local velocity of a scale, $\boldsymbol{u}_{m}(x, y) \equiv\left(u_{m}, v_{m}\right)$, and the local temperature of the scale, $\Theta_{m}(x, y)$, are defined as

$$
\begin{gathered}
\boldsymbol{u}_{m}(x, y)=\left(u_{m}, v_{m}\right) \equiv \sum_{j} \boldsymbol{u}_{m, j}, \\
\Theta_{m}(x, y) \equiv \sum_{j} \Theta_{m, j}
\end{gathered}
$$

Next we define $\Sigma_{m}$ by the spatial average of the larger eigenvalue of the symmetric part of $\nabla^{t}: \boldsymbol{u}_{m}$, because the strain is essential for the dynamics of the $T$ vorticity. The definition is

$$
\Sigma_{m} \equiv \frac{1}{L^{2}} \iint s_{+}(x, y) d x d y,
$$

where $L$ is the period length of the system and

$$
\begin{aligned}
s_{ \pm}(x, y)= & \frac{1}{2}\left\{\left(\partial_{x} u_{m}+\partial_{y} v_{m}\right)\right. \\
& \left. \pm \sqrt{\left(\partial_{x} u_{m}-\partial_{y} v_{m}\right)^{2}+\left(\partial_{x} v_{m}+\partial_{y} u_{m}\right)^{2}}\right\} .
\end{aligned}
$$

It should be noted that $\Sigma_{m}$ is not affected by the term $\left(\partial_{x} u_{m}+\partial_{y} v_{m}\right)$ because this term vanishes when integrated over the space. However, the term $\left(\partial_{x} u_{m}+\partial_{y} v_{m}\right)=\boldsymbol{\nabla} \cdot \boldsymbol{u}_{m}$ itself does not always vanish since an orthonormal wavelet is used to construct $\boldsymbol{u}_{m}$. In fact, in our definitions in terms of an orthonormal wavelet, neither the modal wavelet velocity $\boldsymbol{u}_{m, j}$ nor the local velocity of scale $l_{m}, \boldsymbol{u}_{m}$, is any longer incompressible, while the incompressibility becomes less important for $\Sigma_{m}$. If the spatial distribution of the strain field is analyzed, another definition will be more useful. One possibility is $s_{m}(x, y)$ defined as $\left[s_{+}(x, y)-s_{-}(x, y)\right] / 2$. If $\Sigma_{m}$ were defined by the summation of $s_{m}$ over space, it would be equal to definition (3.5).

In our cascade picture, the $T$ vorticity of a scale $l_{m}$ is related to the entropy of the same scale. Therefore we define $X_{m}$ as a rough estimation of the spatial average of the amplitude of $\left(\partial_{y} \Theta_{m},-\partial_{x} \Theta_{m}\right)$, although we can also use the wavelet transform of $\boldsymbol{\chi}$ itself for $X_{m}$. The concrete definition of $X_{m}$ is

$$
X_{m} \equiv l_{m}^{-1} T_{m},
$$




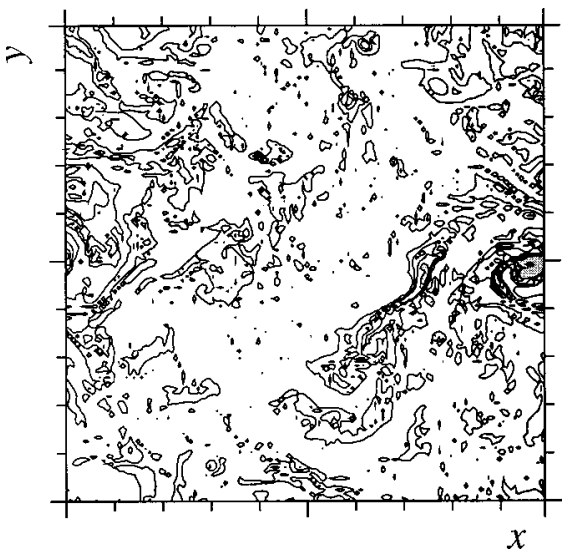

FIG. 1. A snapshot of the entropy field. Contour lines are drawn at $m_{S}, m_{S}+V_{S}^{1 / 2}, m_{S}+2 V_{S}^{1 / 2}$, and $m_{S}+3 V_{S}^{1 / 2}$, where $m_{S}$ is the mean and $V_{S}$ is the variance of the entropy. Regions where the entropy exceeds $m_{S}+3 V_{S}^{1 / 2}$ are shaded.

$$
T_{m} \equiv \frac{1}{L^{2}} \iint\left|\Theta_{m}(x, y)\right| d x d y .
$$

\section{B. Results of DNS}

Direct numerical simulation is carried out with Eqs. (1.1), (1.2), and (1.3) using the fourth-order Runge-Kutta method in integrating the system, and the pseudospectral method in a doubly periodical box, $[0,2 \pi] \times[0,2 \pi]$. The number of modes $N^{2}$ is $256^{2}$. Aliasing terms are removed in terms of the 1/2-shifted grids: thus the effective modes are $(8 / 9) N^{2}$. Hyperviscosity terms $\nu_{H} \Delta^{8} \boldsymbol{u}$ and $\kappa_{H} \Delta^{8} T$ are employed instead of the normal viscosity terms in Eqs. (1.1) and (1.2) to obtain the inertial range clearly. Without loss of generality we can set $\alpha g=1$. Here we also set $\nu_{H}=\kappa_{H}=5 \times 10^{-31}$ and a time step $\Delta t=2.0 \times 10^{-3}$. The forcing term $F$ $=\cos (2 x) \cos (2 y)$ is introduced into Eq. (1.2) to drive the temperature field. The drag term $D=0.5[1-\theta(\mid \boldsymbol{k}$ $-3 \mid)] \Delta^{-1} \boldsymbol{u}$, where $\theta(x)$ is Heviside's step function, is also introduced to Eq. (1.1) to keep the system statistically stationary. It should be noted that a field constituted by $256^{2}$ Fourier modes can be represented by a set of wavelet modes, $\left\{(m, \boldsymbol{j}) \mid \boldsymbol{j}=\left(j_{x}, j_{y}\right) ; 0 \leqslant m \leqslant 8,0 \leqslant j_{x}, j_{y} \leqslant 2^{m}-1\right\}$.

As a preliminary step, we investigate the spatial distribution of entropy briefly. In Fig. 1, we show a snapshot of entropy field at an active time $t=9.2$ when entropy transfer to small scales seems to be enhanced appreciably. In the right of the figure, we can see a strong eddylike structure sprouting linelike structures from it. Although we also observe a corresponding vortex in the vorticity field (not shown in this paper), these vortices are localized even in scale and are not robust, unlike those in 2D NS turbulence. During an active period, eddylike structures become obvious and stretch linelike structures out. It was shown that entropy transfer is enhanced around the region where the linelike structures stretched out [20].

In Fig. 2, we show the normalized histogram for a spatial distribution of wavelet modal entropy, $\sum_{q=1}^{3}\left(\hat{\Theta}_{m, j}^{(q)}\right)^{2} / 2$, for $m=6$. In orthonormal wavelet analysis, the fluctuation due to a phase intrinsic to the wavelet base blurs the observed quantities. Here we eliminate the phase by the method pro-

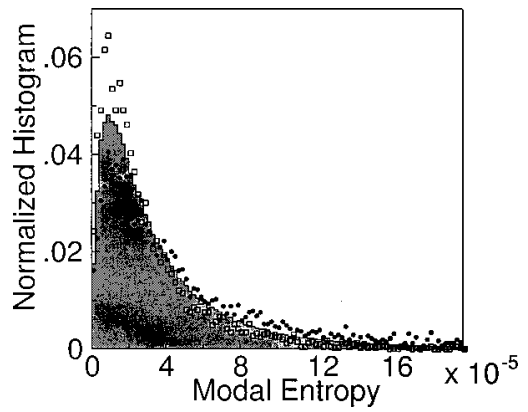

FIG. 2. Normalized histograms of the wavelet entropy within the scale $\sim 2^{-m}$, where $m=6$. The histogram averaged over 60 snapshots is shown shaded. Black circle: most active. Open square: most inactive.

posed in Ref. [21]. The shaded region shows the histogram averaged over 60 snapshots. Squares and black circles show those at the most active and the most inactive times among the 60 samples, respectively. The forms of three histograms are similar. This suggests that activated regions which may correspond to the structures mentioned above are small, and that the rest part is not affected significantly by the active regions. Furthermore, we deduce that the rest part or the background of the structures support most of entropy transfer on average. In this sense, the structures or the active regions seem to contribute to intermittency. In this paper, we focus on the characteristic times related to the entropy transfer process, and so we leave the details of the spatial distribution for future works. In the following, we only deal with variables integrated over a shell.

Now we examine the dynamics due to interactions of the $T$ vorticity and velocity gradient by measuring correlation times between $X_{m}$ and $\Sigma_{m}$. First of all, we compare the magnitudes of the fluctuations of $X_{m}$ and $\Sigma_{m}$ to their mean values to see the relative significance of the fluctuations. In Fig. 3, the average, the standard deviation, and the ratio of the average to the standard deviation for $X_{m}$ and $\Sigma_{m}$ are plotted. If $\Sigma_{m}$ and $X_{m}$ obey BO scaling, the scaling relations in the inertial range are

$$
\left\langle\Sigma_{m}\right\rangle \sim l_{m}^{-2 / 5}
$$
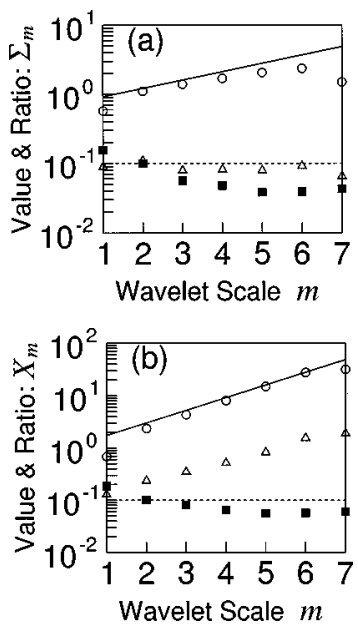

FIG. 3. The average (open circle), the standard deviation (open triangle), and the ratios of the average to the standard deviation (closed square): (a) $X_{m}$, (b) $\Sigma_{m}$. 

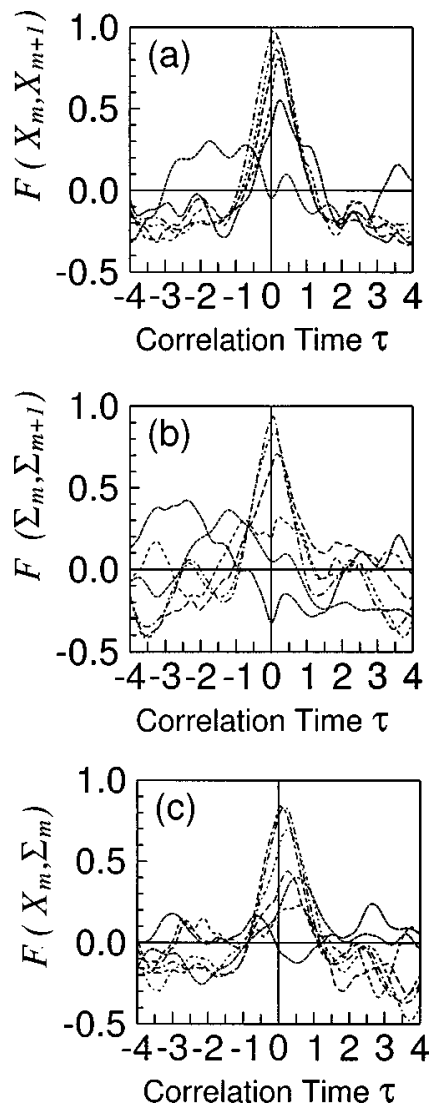

FIG. 4. Cross-correlation functions. Their values at $\tau=0$ increase with $m$, and the top is $m=6$. (a) $F\left(X_{m}, X_{m+1}\right)(\tau)$. (b) $F\left(\Sigma_{m}, \Sigma_{m+1}\right)(\tau)$. (c) $F\left(X_{m}, \Sigma_{m}\right)(\tau)$.

$$
\left\langle X_{m}\right\rangle \sim l_{m}^{-4 / 5}
$$

where \langle\rangle denotes the temporal average. In the range $2 \leqslant m$ $\leqslant 6$, both scalings (3.9) and (3.10) are satisfied. The ratios are less than 0.1 for both $X_{m}$ and $\Sigma_{m}$. Thus the magnitudes of the fluctuations around their mean values are small compared to the averages.

We define the characteristic time of the entropy transfer process as the time at which the cross-correlation coefficient peaks in the same way as that in Ref. [13]. This definition of the characteristic time corresponds to the transfer time, defined in Sec. I as the time required for alternation of generations. Here the cross-correlation coefficient between signals $A(t)$ and $B(t)$ is defined as

$$
F(A, B)(\tau) \equiv \frac{(\langle A(t)-\langle A\rangle\rangle)(\langle B(t+\tau)-\langle B\rangle\rangle)}{\sqrt{\left(\left\langle A^{2}(t)\right\rangle-\langle A\rangle^{2}\right)\left(\left\langle B^{2}(t)\right\rangle-\langle B\rangle^{2}\right)}} .
$$

Figure 4 shows the three correlation functions $F\left(X_{m}, X_{m+1}\right)(\tau), \quad F\left(\Sigma_{m}, \Sigma_{m+1}\right)(\tau)$, and $F\left(X_{m}, \Sigma_{m}\right)(\tau)$. The correlation function $F\left(X_{m}, X_{m+1}\right)(\tau)$ shows that $X_{m}$ and $X_{m+1}$ correlate well: the function attains a maximum value larger than 0.8 for $m \geqslant 3$. The sign of the time at which $F\left(X_{m}, X_{m+1}\right)(\tau)$ peaks is positive for $m \geqslant 2$. Thus $X_{m}$ or, equivalently, the entropy is transferred to smaller scales in the inertial range.

The correlation function $F\left(\Sigma_{m}, \Sigma_{m+1}\right)(\tau)$ also attains a maximum value larger than 0.8 for $m \geqslant 4$. The sign of the

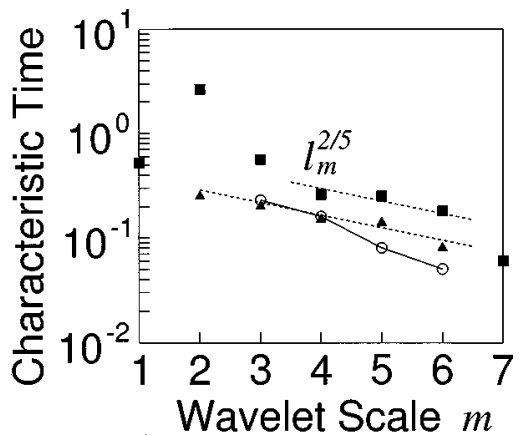

FIG. 5. Correlation times of $F\left(X_{m}, X_{m+1}\right)(\tau)$, $F\left(\Sigma_{m}, \Sigma_{m+1}\right)(\tau)$, and $F\left(X_{m}, \Sigma_{m}\right)(\tau)$. The lines show the BO time scaling.

time at which $F\left(\Sigma_{m}, \Sigma_{m+1}\right)(\tau)$ peaks is positive for $m \geqslant 3$. However, unlike the case of $X_{m}$, this result is not necessarily explained by the nonlinear interaction among $\Sigma_{m}$, because the buoyancy dominates the nonlinear interaction in the early stage of the excitation of $\Sigma_{m}$. The width of $F\left(\Sigma_{m}, \Sigma_{m+1}\right)(\tau)$ is about the same as that of $F\left(X_{m}, X_{m+1}\right)(\tau)$ for $m \geqslant 4$.

In Ref. [13], a similar analysis was applied for the cumulative wavelet energy and entropy within a scale. The result in Ref. [13] is that $F\left(E_{m}, E_{m+1}\right)(\tau)$ takes two local maxima around $\tau=0$, where $E_{m}$ is the cumulative modal wavelet energy within a scale $l_{m}$. It has been concluded that the kinetic energy transfer does not take a definite direction unlike the entropy transfer. Because $F\left(\Sigma_{m}, \Sigma_{m+1}\right)(\tau)$ seems to have a single maximum, the negative energy transfer is not detected clearly by $\Sigma_{m}$. Thus the velocity gradient $\Sigma_{m}$ is more convenient than $E_{m}$ when we focus on the entropy transfer. It should be noted that energy is transferred inversely on average, and this inverse transfer is crucial for the BO scaling to dominate the Kolmogorov scaling.

The maximum value of the function $F\left(X_{m}, \Sigma_{m}\right)(\tau)$ is not as large as that of $F\left(X_{m}, X_{m+1}\right)(\tau)$ or $F\left(\Sigma_{m}, \Sigma_{m+1}\right)(\tau)$. The maximum is at most 0.8 for $m=6$ and 7 , and less than 0.7 for $m \leqslant 5$. This may be explained as follows. There seem to be at least two processes for the transfer of $\Sigma_{m}$, which is expected by the two terms on the right-hand side of Eq. (2.1): one is the induction of $X_{m}$ by $\Sigma_{m}$ due to the buoyancy effect, and the other is the interaction among $\Sigma_{m}$. Because the latter does not seem to relate directly to the former, i.e., $X_{m}$, the cross-correlation between $\Sigma_{m}$ and $X_{m}$ is lower than that between $\Sigma_{m}$ and $\Sigma_{m+1}$, or that between $X_{m}$ and $X_{m+1}$.

The characteristic times of the transfers from $X_{m}$ to $X_{m+1}$, from $\Sigma_{m}$ to $\Sigma_{m+1}$ and from $X_{m}$ to $\Sigma_{m}$ are referred to as $\tau_{m}^{D N S}(X), \tau_{m}^{D N S}(\Sigma)$, and $\tau_{m}^{D N S}(X \rightarrow \Sigma)$, respectively. Each of them is defined as the time at which the correlation function attains a maximum value. In Fig. 5, the characteristic times $\tau_{m}^{D N S}(X), \tau_{m}^{D N S}(\Sigma)$, and $\tau_{m}^{D N S}(X \rightarrow \Sigma)$ are shown. In the range $1 \leqslant m \leqslant 6$, the induction time $\tau_{m}^{D N S}(X \rightarrow \Sigma)$ is more than two times larger than $\tau_{m}^{D N S}(X)$ and $\tau_{m}^{D N S}(\Sigma)$. As far as a single sequence of entropy transfer through the inertial range is concerned, nonlinear interactions among $\Sigma_{m}$ seem to be less important. The fact that $\tau_{m}^{D N S}(X \rightarrow \Sigma)>\tau_{m}^{D N S}(X)$ suggests that the interaction among $\Sigma_{m}$ dominates after the excitation of $X_{m+1}$, while $X_{m+1}$ is excited by the interaction between $\Sigma_{m}$ and $X_{m}$. Therefore $\Sigma_{m}$ seems to be induced 

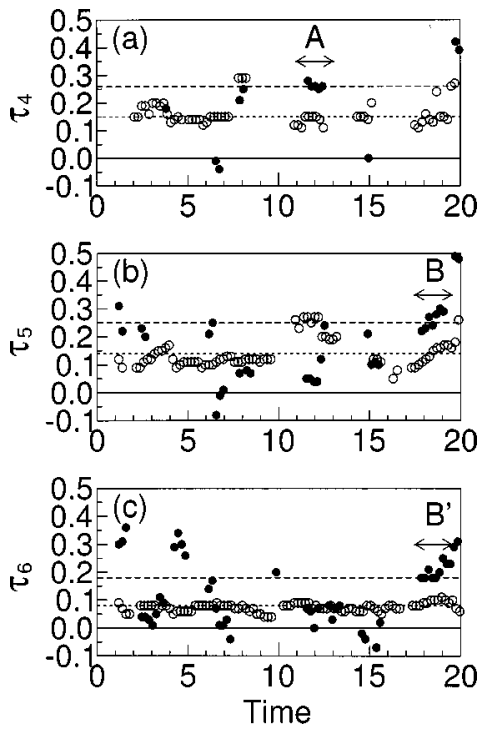

FIG. 6. (a) $\tau\left(X_{m}, X_{m+1}, T_{c}\right)$ as a function of $T_{c}$. (b) $\tau\left(\Sigma_{m}, \Sigma_{m+1}, T_{c}\right)$ as a function of $T_{c}$.

only by the buoyancy in an early stage.

Next we discuss the scaling relations of the characteristic times. Two broken lines representing the BO time scaling $\sim l_{m}^{2 / 5}$, are drawn in Fig. 5 for reference. The lines agree with $\tau_{m}^{D N S}(X)$ and $\tau_{m}^{D N S}(X \rightarrow \Sigma)$ in the range $3<m<6$, but $\tau_{m}^{D N S}(\Sigma)$ differs from the lines. If $\tau_{m}^{D N S}(\Sigma)$ obeys a power law, the exponent is smaller than that of the BO time scaling $-2 / 5$. In our simulation, the number of the modes adopted is not as large that the inertial range observed is not sufficiently wide. In Sec. IV, we show that our shell model indicates the similar tendency of $\tau_{m}^{D N S}(\Sigma)$ for smaller shell numbers. Therefore, it is inferred that the disagreement between the BO time scaling and $\tau_{m}^{D N S}(\Sigma)$ is due to the narrow inertial range.

To examine a period when the entropy transfer process is activated, we define a temporally local correlation time $\tau\left(A, B, T_{c}\right)$ as the time at which the following correlation function attains a local maximum value:

$$
F_{l}(A, B)\left(T_{c}, T_{w}, \tau\right) \equiv \frac{\left(\left\langle A(t)-\langle A\rangle_{l}\right\rangle_{l}\right)\left(\left\langle B(t+\tau)-\langle B\rangle_{l}\right\rangle_{l}\right)}{\sqrt{\left(\left\langle A^{2}(t)\right\rangle_{l}-\langle A\rangle_{l}^{2}\right)\left(\left\langle B^{2}(t)\right\rangle_{l}-\langle B\rangle_{l}^{2}\right)}}
$$

In this definition, $\langle *\rangle_{l}$ denotes the local time average over the period $T_{w}$ centered at $T_{c}$, i.e., $\left(1 / T_{w}\right) \int_{T c-T w / 2}^{T c+T w / 2} * d t$. Here we choose $T_{w}$ as two unit times, which is the width of the cross-correlation functions shown in Figs. 4(a), 4(b), and 4(c).

In Fig. 6, $\tau\left(X_{m}, X_{m+1}, T_{c}\right)$ and $\tau\left(X_{m}, \Sigma_{m}, T_{c}\right)$ are plotted for $m=4,5$, and 6 , when their correlation coefficients are larger than 0.8. The number of points plotted in the figures for $\tau\left(X_{m}, \Sigma_{m}, T_{c}\right)$ is less than that for $\tau\left(X_{m}, X_{m+1}, T_{c}\right)$. This means that the local correlation between $X_{m}$ and $X_{m+1}$ is higher than that between $X_{m}$ and $\Sigma_{m}$. The maximum value of $F\left(X_{m}, X_{m+1}\right)(\tau)$ is larger than that of $F\left(X_{m}, \Sigma_{m}\right)(\tau)$ for $m=4,5$, and 6 . The number of points increases with $m$, since the maximum values of $F\left(X_{m}, X_{m+1}\right)(\tau)$ and $F\left(X_{m}, \Sigma_{m}\right)(\tau)$ becomes larger as $m$ goes large.
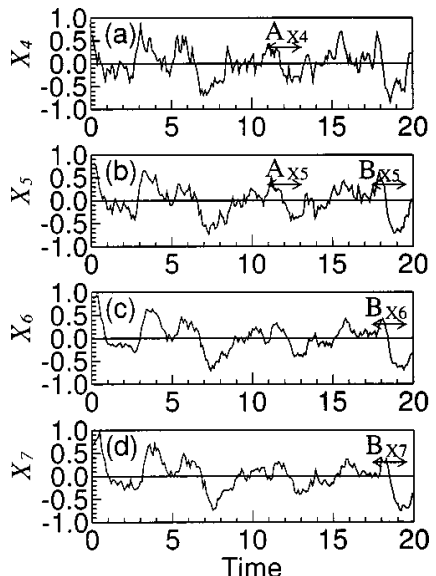

FIG. 7. Time series of $X_{m}(t)$ for $m=4,5$, and 6. Each function is normalized by $3 V_{m}^{1 / 2}$, where $V_{m}$ is the variance of each function.

It is quite interesting that in the several periods, $\tau\left(X_{m}, X_{m+1}, T_{c}\right)$ approaches a constant value as $m$ becomes large, and this constant is the correlation time $\tau_{m}^{D N S}(X)$. This suggests that there exist the well-separated entropy transfer processes. Some of them actually correspond to the entropy cascade picture, because the induction time also obeys the BO time scaling in these special periods. We call these processes characteristic entropy transfer processes. On the other hand, we have deduced that multiprocesses constitute the excitation of $\Sigma_{m}$ from the fact that the correlation $F\left(X_{m}, \Sigma_{m}\right)$ is lower than that of $F\left(X_{m}, X_{m+1}\right)$ and $F\left(\Sigma_{m}, \Sigma_{m+1}\right)$ as shown in Figs. 4(a), 4(b), and 4(c). Thus heavy scattering of $\tau\left(X_{m}, \Sigma_{m}, T_{c}\right)$ seems to be caused by the competition of these multiprocesses, one of which is the buoyancy effect and dominates others on average.

We examine the characteristic entropy transfer processes individually. Some of them are labeled $A, B$, and $B^{\prime}$ where $\tau\left(X_{m}, X_{m+1}, T_{c}\right) \simeq \tau_{m}^{D N S}(X)$ and $\tau\left(X_{m}, \Sigma_{m}, T_{c}\right) \simeq \tau_{m}^{D N S}(X$ $\rightarrow \Sigma)$. These periods are good samples of characteristic dynamical processes corresponding to the entropy cascade picture. We discuss qualitative features of the transfers of the $T$ vorticity and velocity gradient, observing the temporal evolution of $X_{m}(t)$ and $\Sigma_{m}(t)$ shown in Figs. 7 and 8 . The capital letters such as $A_{\Sigma 4}$, etc. marked in Figs. 7 and 8
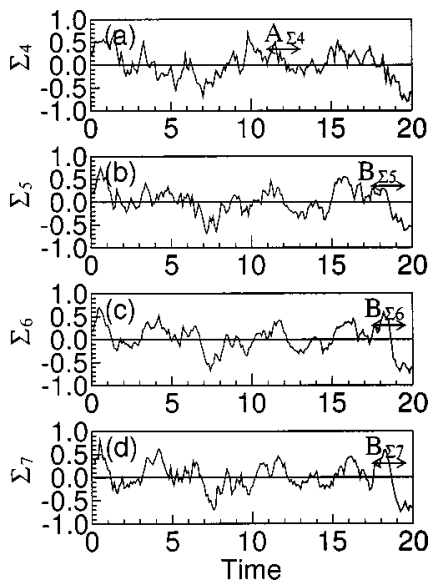

FIG. 8. Same as Fig. 8, except for $\Sigma_{m}(t)$. 
represent the characteristic periods mentioned above. In Figs. 7 and 8 , the symbol $B^{\prime}$ is replaced by $B$ because they represent the same sequence of the transfer. The shapes of $X_{m}(t)$ and $\Sigma_{m}(t)$, belonging to period $B$, are almost the same, but they are different from those in period $A$. We focus on period $B$, where the fluctuations in periods $B_{X 5}, B_{X 6}$, and $B_{X 7}$ correlate highly to each other. The widths of the fluctuations are almost the same, and about two unit times for $B_{X 5}, B_{\Sigma 5}$, $B_{X 6}, B_{\Sigma 6}$, and $B_{X 7}$. If the staying time of a scale, i.e., the width of $B_{X m}$, obeyed the BO time scaling, it would become shorter as $m$ becomes larger and the ratio between the widths of $B_{X 7}$ and $B_{X 5}$ would be $\left(2^{-2 / 5}\right)^{2} \sim 0.57$. Thus we conclude that the staying times for $X_{m}$, or $\Sigma_{m}$ do not satisfy the BO time scaling: the staying time is different from the transfer time defined as the time between the peaks of $X_{m}$ and $X_{m+1}$.

The characteristic entropy transfer processes we examined above differ from the traditional cascade picture with respect to the characteristic times. However, we have introduced another cascade picture with multigeneration, and this picture is rather appropriate for describing these characteristic processes. We will show in Sec. II that these processes are well explained by the shell model.

\section{A SHELL MODEL}

The shell model proposed in Ref. [22] is governed by the equations

$$
\begin{gathered}
\frac{d \sigma_{m}}{d t}=\alpha g \chi_{m}-\sigma_{m}^{2}-\nu \lambda^{2 m} \sigma_{m}+F_{D}(m), \\
\frac{d \chi_{m}}{d t}=\lambda^{2} \sigma_{m-1} \chi_{m-1}-\sigma_{m} \chi_{m+1}-\kappa \lambda^{2 m} \chi_{m}+F_{F}(m),
\end{gathered}
$$

where $\chi_{m}$ and $\sigma_{m}$ are positive variables, and represent the amplitudes $\boldsymbol{\chi}$ and $\boldsymbol{\sigma}$ of scale $l_{m} \equiv \lambda^{-m}$ ( $\lambda$ is the ratio between the length scales of adjacent shells), respectively. The shell number $m$ roughly corresponds to the logarithm of the wave number $\sim 1 / l_{m}$.

We have derived these model equations according to the entropy cascade picture described in Sec. II. Since the energy is transferred inversely on average in DNS's, the nonlinear effects in a scale are weak before the modes of a smaller scale are excited sufficiently. Thus the model equation for $\sigma_{m}$ is dominated by the buoyancy term. Furthermore, we replace the transfer of kinetic energy with the nonlinear saturation term $-\sigma_{m}^{2}$, and then Eq. (4.1) is closed in a shell. This nonlinear term also allows steady solutions satisfying the BO scalings $\sigma_{m} \sim \lambda^{2 m / 5}$ and $\chi_{m} \sim(\alpha g)^{-1} \lambda^{4 m / 5}$ in the inertial range. Convective terms and pressure terms are neglected for simplicity. The drag term $F_{D}(m)=-D\left(\delta_{m, 1} / \lambda+\delta_{m, 2} / \lambda^{2}\right.$ $\left.+\delta_{m, 3} / \lambda^{3}\right)$ is introduced to prevent the variables from diverging.

The model equation for $\chi_{m}$ is essentially the same as that of $T_{m}$ in the original shell model, which causes an entropy cascade, as seen by replacing $T_{m}$ and $u_{m}$ with $l_{m} \chi_{m}$ and $l_{m} \sigma_{m}$, respectively $[18,19]$. The localness of nonlinear interactions is assumed as in typical shell models. Thus the total entropy $\Sigma_{m} T_{m}^{2} / 2$ is conserved in the inviscid case. The forcing term $F_{F}(m)=F \delta_{m, 4}$ is introduced to allow the system to

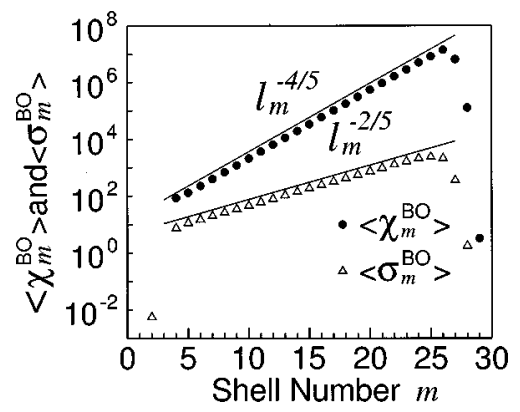

FIG. 9. The stable solutions $\left\langle\chi_{m}^{\mathrm{BO}}\right\rangle$ and $\left\langle\sigma_{m}^{\mathrm{BO}}\right\rangle$. The lines show the $\mathrm{BO}$ scaling.

sustain a steady solution. It should be noted that our shell model is not a chaotic system, unlike the original shell model.

\section{A. BO case}

We have introduced our shell model to examine each of the characteristics of entropy transfer processes observed in DNS's which seem to be candidates for elementary processes of entropy cascade. Through each of these transfer processes, the modes $\Sigma_{m}$ and $X_{m}$ constituting fluctuations are highly correlated to each other, i.e., these processes can be regarded as dynamical ones. Thus we believe that chaotic behavior is not required for our purpose. The fluctuations in these processes are small when compared to the background field, i.e., the statistically quasiequilibrium state. Then we first obtain steady solutions of our shell model obeying the BO scaling in the inertial range, and observe the temporal evolution of a fluctuation added to these steady solutions at a large scale.

Simulations of our shell model are performed using the fourth-order Runge-Kutta method. We set $\lambda=2$ hereafter. The steady solutions are obtained for $F=D=1.0 \times 10^{3}, \nu$ $=\kappa=1.0 \times 10^{-12}$, and $\alpha g=1$. These steady solutions, referred to as $\left\langle\sigma_{m}^{\mathrm{BO}}\right\rangle$ and $\left\langle\chi_{m}^{\mathrm{BO}}\right\rangle$, obey the BO scaling in the range between $10<m<20$, as seen in Fig. 9. We have called this range the inertial range of the steady solutions for convenience. The initial condition was constructed by adding a small fluctuation $\delta \chi=1.0 \times 10^{-2}$ at $m=4$ to $\left\langle\chi_{m=4}^{\mathrm{BO}}\right\rangle$ in surplus. We refer to this situation as the $\mathrm{BO}$ case. The solutions, referred to as $\sigma_{m}^{\mathrm{BO}}(t)$ and $\chi_{m}^{\mathrm{BO}}(t)$, are obtained with $\Delta t$ $=1.0 \times 10^{-6}$.

We examine the $\mathrm{BO}$ case, focusing particularly on characteristic times: the transfer time and the staying time. Here we introduce the characteristic times of a mode $m, \tau_{m}^{\sigma}, \tau_{m}^{\chi}$, and $\tau_{m}^{\chi \rightarrow \sigma}$, which are defined as $\tau_{m}^{\sigma}=t_{m+1}^{\sigma}-t_{m}^{\sigma}, \tau_{m}^{\chi}=t_{m+1}^{\chi}$ $-t_{m}^{\chi}$, and $\tau_{m}^{\chi \rightarrow \sigma}=t_{m}^{\sigma}-t_{m}^{\chi}$, respectively. In these definitions, $t_{m}^{\sigma}$ and $t_{m}^{\chi}$ denote the times at which $\sigma_{m}$ and $\chi_{m}$ attain their maximum values, respectively.

In Fig. 10, $\tau_{m}^{\sigma}, \tau_{m}^{\chi}$, and $\tau_{m}^{\chi \rightarrow \sigma}$ are plotted as functions of $m$. In the range $10<m<20$, all of them obey the BO time scaling. In the range $5 \leqslant m \leqslant 10, \tau_{m}^{\chi}$ and $\tau_{m}^{\chi \rightarrow \sigma}$ satisfy the BO time scaling, but $\tau_{m}^{\sigma}$ decreases faster than the BO time scaling as $m$ increases. This behavior of the characteristic times is reminiscent of the results of DNS shown in Fig. 5. This suggests that the discrepancy of $\tau_{m}^{D N S}(\Sigma)$ from the BO scaling is due to the short inertial range. 


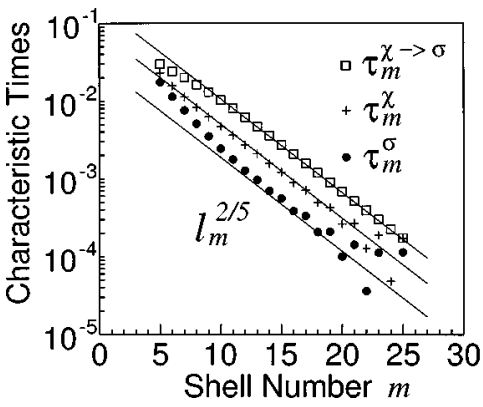

FIG. 10. The characteristic times of $\sigma_{m}$ and $\chi_{m}$ in the BO case. The lines show the BO time scaling.

As shown in Fig. 11, functions $\sigma_{m}^{\mathrm{BO}}(t)$ and $\chi_{m}^{\mathrm{BO}}(t)$ of the modes in the inertial range coincide with the same shape under a proper normalization: if we define the normalized time $\tau_{m}$ and $\tau_{m}^{\prime}$ as $\tau_{m} \equiv\left(t-t_{m}^{\sigma}\right) / t_{m}^{\sigma}$ and $\tau_{m}^{\prime} \equiv\left(t-t_{m}^{\chi}\right) / t_{m}^{\chi}$, respectively, we obtain the two similar functions $S_{m}\left(\tau_{m}\right)$ $\equiv\left[\sigma_{m}^{\mathrm{BO}}(t)-\left\langle\sigma_{m}^{\mathrm{BO}}\right\rangle\right] /\left\langle\sigma_{m}^{\mathrm{BO}}\right\rangle \quad$ and $\quad K_{m}\left(\tau_{m}^{\prime}\right) \equiv\left[\chi_{m}^{\mathrm{BO}}(t)\right.$ $\left.-\left\langle\chi_{m}^{\mathrm{BO}}\right\rangle\right] /\left\langle\chi_{m}^{\mathrm{BO}}\right\rangle$ where $S_{m}$ and $K_{m}$ are independent of $m$. If $l_{m}$ is in the inertial range, i.e., $m$ is large, the normalization factor $t_{m}^{\chi}$ or $t_{m}^{\sigma}$ is almost constant because $\tau_{m}^{\chi} \ll t_{m}^{\chi}$ and $\tau_{m}^{\sigma}$ $\ll t_{m}^{\sigma}$, respectively. Thus the introduction of the normalized times $\tau_{m}$ and $\tau_{m}^{\prime}$ virtually means the translation of the origin. This is consistent with the result of DNS's. In Fig. 7, the fluctuations $B_{X 5}, B_{X 6}$, and $B_{X 7}$ have almost the same shape, although the ratio of the scale of $B_{X 7}$ to that of $B_{X 5}$ is $1 / 4$. This similarity of $S_{m}$ and $K_{m}$ is well explained by perturbation analysis in Sec. V.

These results mean that we should introduce two characteristic times for the entropy transfer, as mentioned in Sec. I: the transfer time and the staying time. The transfer time, such as $\tau_{m}^{\chi}$, is the time in which fluctuations are transferred from a shell $m$ to $m+1$. The staying time of a fluctuation, which is of order $t_{m}^{\chi}$, is the time in which a fluctuation of a scale $m$ loses its appreciable part. In the BO case, only the transfer time obeys the BO time scaling. On the other hand, the staying time is almost the same in the inertial range. The result is contradictory to the traditional cascade picture in which there is only one characteristic time. The BO case rather corresponds to the multigeneration cascade picture introduced in Sec. I. It should be noted that we extend the meaning of the transfer time to express the transfer processes of nonconserved quantities, such as $\sigma_{m}$ and $\chi_{m}$.

In 3D NS turbulence [14], the transfer time was examined indirectly by observing the fluctuations of transfer function. It is shown that the transfer time does not contradict the Kolmogorov time scaling. However, the staying time has not been examined in 3D NS turbulence. Therefore, we cannot conclude whether the difference of these two characteristic times is common in 2D FC and 3D NS turbulences or not. In Sec. V, it is proved analytically that the transfer time satisfies the BO time scaling. In Appendix B, it is shown that the original shell model for FC and shell models for NS have the same similarity as our model. Thus we suggest that the NS system has two different characteristic times when the transfer of fluctuations around the steady solution or the statistical quasiequilibrium state, i.e., the Kolmogorov spectrum, is considered.
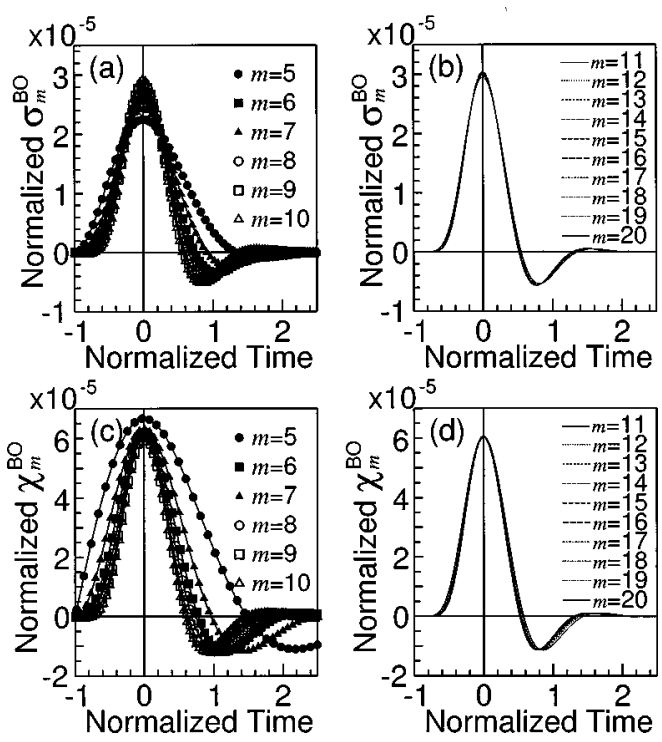

FIG. 11. Temporal evolutions of $S_{m}\left(\tau_{m}\right)$ and $T_{m}\left(\tau_{m}^{\prime}\right)$ in the BO case. $S_{m}\left(\tau_{m}\right)$ : (a) for $m=5,6, \ldots, 10$, and (b) in the inertial range. $T_{m}\left(\tau_{m}^{\prime}\right)$ : (c) for $m=5,6, \ldots, 10$, and (d) in the inertial range.

\section{B. Blast case}

Next, we investigate another situation where the system is far from the quasiequilibrium state initially. That is, we treat the response of the system in a null state to the injection of the entropy of large scale. This case is called the blast case hereafter. The time-dependent solution describing the response shows that the maximum value of each mode satisfies different scaling relations from the $\mathrm{BO}$ scaling. We call the spectrum of the maximum values the peak spectrum hereafter. The peak spectra for the BO case satisfy the BO scaling, which is trivial due to the similarity of $S_{m}$ and $K_{m}$ shown in Sec. IV A.

In this case, all modes are set null except that $\chi_{m=4}$ $=1.0$ in the initial condition. The time step $\Delta t$ is 1.0 $\times 10^{-6}$, the total number of steps is $3 \times 10^{6}, \nu=\kappa=1.0$ $\times 10^{-12}$, and $F=D=0$. These solutions obtained by the simulation are referred to as $\sigma_{m}^{\mathrm{bl}}(t)$ and $\chi_{m}^{\mathrm{bl}}(t)$.

In the blast case, if we define the normalized time $\eta_{m}$ and $\eta_{m}^{\prime}$ as $\eta_{m} \equiv\left(t-t_{m}^{\sigma}\right) / \tau_{m}^{\sigma}$ and $\eta_{m}^{\prime} \equiv\left(t-t_{m}^{\chi}\right) / \tau_{m}^{\chi}$, the functions $S_{m}\left(\eta_{m}\right) \equiv \sigma_{m}^{\mathrm{bl}}(t) /\left[\sigma_{m}^{\mathrm{bl}}\right]$ and $T_{m}\left(\eta_{m}^{\prime}\right) \equiv \chi_{m}^{\mathrm{bl}}(t) /\left[\chi_{m}^{\mathrm{bl}}\right]$ are almost the same, where $\left[\sigma_{m}^{\mathrm{bl}}\right]$ and $\left[\chi_{m}^{\mathrm{bl}}\right]$ are the peak spectra (Fig. 12). It should be noted that the definitions of $\eta_{m}$ and $\eta_{m}^{\prime}$ are different from the counterparts in the $\mathrm{BO}$ case.

The peak spectra and the characteristic times, defined in the same way as in the BO case satisfy the different scaling relations from the $\mathrm{BO}$ scaling. The scalings for the blast case are

$$
\begin{gathered}
{\left[\sigma_{m}^{\mathrm{bl}}\right] \sim(\alpha g)^{1 / 2} S_{0}^{1 / 4} \lambda^{(1 / 2) m},} \\
{\left[\chi_{m}^{\mathrm{bl}}\right] \sim S_{0}^{1 / 2} \lambda^{m},} \\
\tau_{m}^{\mathrm{bl}} \sim(\alpha g)^{-1 / 2} S_{0}^{-1 / 4} \lambda^{-(1 / 2) m},
\end{gathered}
$$

where $S_{0}$ is the total entropy injected initially. Since the time is normalized by $\tau_{m}^{\sigma}$ or $\tau_{m}^{\chi}$, the staying time is also scaled by Eq. (4.5) in this case. Hereafter we call scalings (4.3) and 

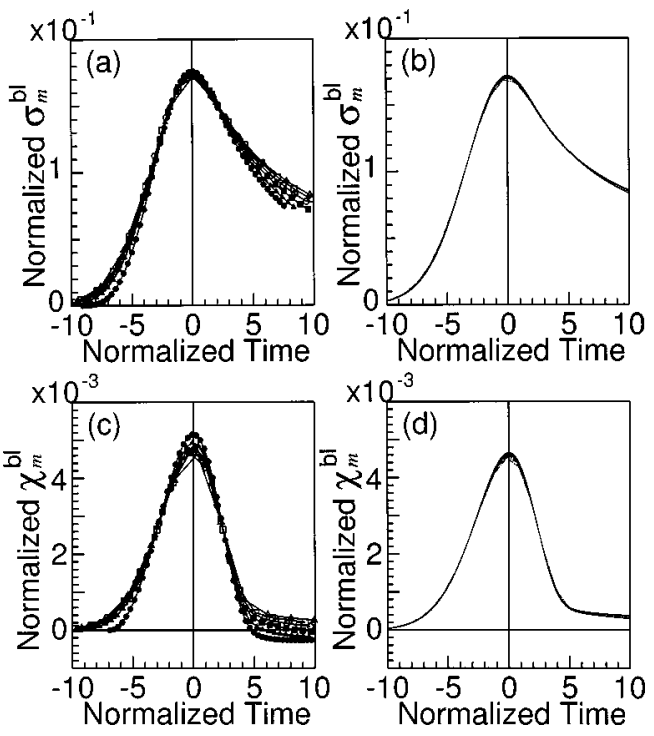

FIG. 12. Temporal evolutions of $S_{m}^{b l}\left(\tau_{m}\right)$ and $T_{m}^{b l}\left(\tau_{m}^{\prime}\right)$ in the blast case. $S_{m}^{b l}\left(\eta_{m}\right)$ : (a) for $m=5,6, \ldots, 10$, and (b) in inertial range. $T_{m}^{b l}\left(\eta_{m}^{\prime}\right)$ : (c) for $m=5,6, \ldots, 10$, and (d) in inertial range.

(4.4) the blast peak spectra. The scaling relation (4.5) has been confirmed for different values of $S_{0}$ and $\alpha g$. In Fig. 13, the normalized time scales $\tau_{m}(\alpha g)^{1 / 2} S_{0}^{1 / 4}$ for the induction time $\tau_{m}^{\chi \rightarrow \sigma}$ are plotted. It is scaled as $\sim l_{m}^{1 / 2}$.

In Fig. 14, the (normal) spectra for $\chi_{m}$ and $\sigma_{m}$ are shown at several times. One spectrum obtained after the time when the blast reaches the dissipation range is also shown. Before the blast reaches the dissipation range, its scaling is different from the BO scaling. In the case of $\chi_{m}$, it does not satisfy even the scaling relation, while $\sigma_{m}$ seems to obey a power law but its exponent varies as time goes on. On the other hand, the maximum values of $\sigma_{m}$ and $\chi_{m}$ obey scalings (4.3) and (4.4) which are drawn as bold straight lines in Figs. 13(a) and 13(b), respectively. This means that even if the blast dominates the system, the BO spectra will be retrieved after the blast reaches the dissipation range. In fact, this time is finite and evaluated as follows: $t_{\infty} \equiv \sum_{m=0}^{\infty} \tau_{m}^{\mathrm{bl}}$ $=(\alpha g)^{-1 / 2} S_{0}^{-1 / 4}\left(1-\lambda^{-1 / 2}\right)^{-1}<\infty$.

It should be noted that the staying time and the transfer time are identical in this case. In this sense, the blast case is rather similar to the traditional cascade picture. However, we do not use the average dissipation rate of the entropy, but the total entropy in the dimensional analysis, to derive scalings (4.3), (4.4), and (4.5) [22]. Even in the blast case, the entropy flux averaged over $t_{\infty}$ is independent of $m$. At the moment, we can only point out that the blast case may be one candidate for an elementary process of entropy cascade; however, we do not claim further significance of the blast case here.

The essential difference between the $\mathrm{BO}$ case and the blast case is the ratio of the entropy injected in the initial condition to the entropy of the background states. Thus it is suggested that when the strength of the initial fluctuation of the BO case becomes sufficiently large, the fluctuation dominates the steady solutions and obeys the scalings for the blast case. Here we examine the dependence of the $\mathrm{BO}$ case on the strength of the initial fluctuation, to see where and how the BO peak spectra are converted into the blast peak spectra. As seen in Fig. 15, a significant transition from the BO peak
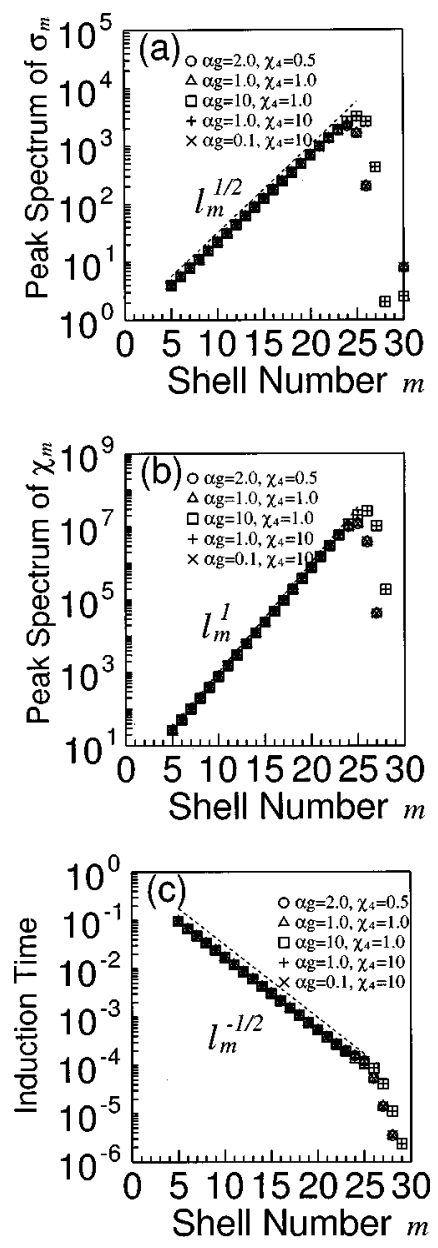

FIG. 13. Normalized peak spectra and the time scaling for several values of the set $\left(S_{0}, \alpha g\right)$ : (a) $\left[\sigma_{m}^{b l}\right](\alpha g)^{-1 / 2} S_{0}^{-1 / 4}$, (b) $\left[\chi_{m}^{b l}\right] S_{0}^{-1 / 2}$, and (c) $\tau_{m}^{b l}$.

spectra to the blast peak spectra occurs between $\delta \chi_{4}=2$ $\times 10^{3}$ and $\delta \chi_{4}=5 \times 10^{3}$. The rough sketch of the transition is as follows. The transition starts with the appearance of the blast peak spectra in a small shell. After that the crossover moves to a larger shell as the value of $\delta \chi_{4}$ increases. Finally, the entire range is occupied by the blast peak spectra. It should be noted that the transition is not so clear, and the spectra do not obey the scalings for the BO and the blast cases strictly for intermediate values of $\delta \chi_{4}$.

\section{PERTURBATION ANALYSIS}

In Sec. IV, numerical simulations revealed that $\sigma_{m}(t)$ and $\chi_{m}(t)$ have almost the same shape in the inertial range if properly normalized. Here, by perturbation analysis, we try to explain the similarity of $\sigma_{m}(t)$ and $\chi_{m}(t)$ for the BO case. Through this analysis, characteristic times are derived consistently. In Appendix B, a similar analysis is applied for a class of shell models for NS, and the original shell model for free convection, to show that these shell models can be included in the same class as ours.

We introduce the nondimensionalized similar solutions of $\sigma_{m}(t)$ and $\chi_{m}(t)$ :

$$
\epsilon S(\tau) \equiv \frac{\sigma_{m}(t)-\left\langle\sigma_{m}^{\mathrm{BO}}\right\rangle}{\left\langle\sigma_{m}^{\mathrm{BO}}\right\rangle},
$$



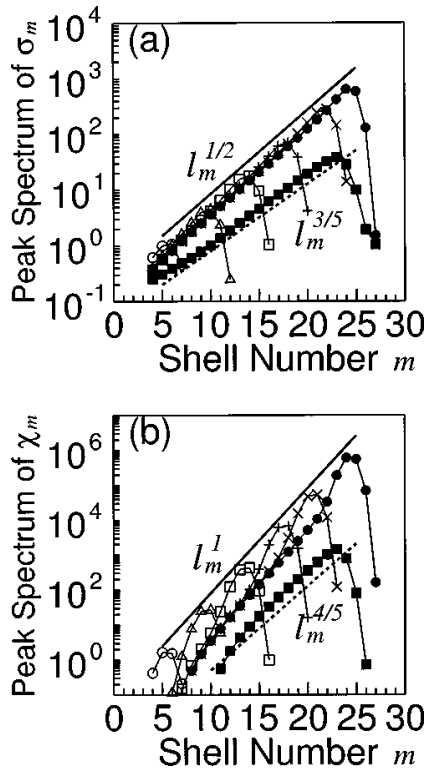

FIG. 14. Temporal evolution of the spectra of the blast case. Lines are plotted at times in which each mode peaks, and one time after the blast reaches to the dissipation range. (a) $\sigma_{m}^{b l}(t)$. (b) $\chi_{m}^{b l}(t)$.

$$
\epsilon K\left(\tau^{\prime}\right) \equiv \frac{\chi_{m}(t)-\left\langle\chi_{m}^{\mathrm{BO}}\right\rangle}{\left\langle\chi_{m}^{\mathrm{BO}}\right\rangle}
$$

where the small parameter $\epsilon$ indicates the order of the relative strength of the fluctuation with regard to the steady solution. Thus $S$ and $K$ are of order 1 . The normalized times $\tau$ and $\tau^{\prime}$ are also defined on a particular shell $m$ as $\tau \equiv(t$ $\left.-t_{m}^{\sigma}\right) / t_{m}^{\sigma}$ and $\tau^{\prime} \equiv\left(t-t_{m}^{\chi}\right) / t_{m}^{\chi}$. Introducing another small parameter $\epsilon_{m} \equiv\left(t_{m}^{\sigma}\left\langle\sigma_{m}^{\mathrm{BO}}\right\rangle\right)^{-1}$, and substituting the similar solutions (5.1) and (5.2) to Eq. (4.1) with $D=\nu=\kappa=0$, we obtain the equation

$$
\epsilon \epsilon_{m} \frac{d S}{d \tau}=\epsilon\left\{K\left(\tau^{\prime}\right)-2 S(\tau)\right\}-\epsilon^{2} S^{2}(\tau),
$$

where the existence of $S$ and $K$ are assumed.

We request that the sums of characteristic times converge at the same finite value as $m$ tends to infinity:

$$
\lim _{m \rightarrow \infty} t_{m}^{\sigma}=\lim _{m \rightarrow \infty} t_{m}^{\chi}=t_{\infty}<\infty
$$

Then $\lim _{m \rightarrow \infty} \epsilon_{m}=1 /\left(t_{m}^{\sigma}\left\langle\sigma_{m}^{\mathrm{BO}}\right\rangle\right)=0$ because $\left\langle\sigma_{m}^{\mathrm{BO}}\right\rangle \sim \lambda^{3 m / 5}$ and $\lambda>1$. Condition (5.4) also means that $\lim _{m \rightarrow \infty} \tau_{m}^{\sigma}$ $=0, \lim _{m \rightarrow \infty} \tau_{m}^{\chi}=0$, and $\lim _{m \rightarrow \infty} \tau_{m}^{\chi \rightarrow \sigma}=0$. We focus on the period $|\tau| \ll 1$, where

$$
\begin{aligned}
\tau^{\prime}= & \tau+\frac{\tau_{m}^{\chi \rightarrow \sigma}}{t_{m}^{\sigma}}+O\left(\left(\frac{\tau_{m}^{\chi \rightarrow \sigma}}{t_{m}^{\sigma}}\right)^{2}\right)=\tau+\epsilon_{m} \tau_{m}^{\chi \rightarrow \sigma}\left\langle\sigma_{m}^{\mathrm{BO}}\right\rangle \\
& +O\left(\left(\epsilon_{m} \tau_{m}^{\chi \rightarrow \sigma}\left\langle\sigma_{m}^{\mathrm{BO}}\right\rangle\right)^{2}\right),
\end{aligned}
$$

because $t_{m}^{\sigma}=t_{m}^{\chi}+\tau_{m}^{\chi \rightarrow \sigma}$. Then we can evaluate $K\left(\tau^{\prime}\right)$ as

$$
K\left(\tau^{\prime}\right)=K(\tau)+\epsilon_{m} \tau_{m}^{\chi \rightarrow \sigma}\left\langle\sigma_{m}^{\mathrm{BO}}\right\rangle \frac{d K}{d \tau}+O\left(\left(\epsilon_{m} \tau_{m}^{\chi \rightarrow \sigma}\left\langle\sigma_{m}^{\mathrm{BO}}\right\rangle\right)^{2}\right)
$$

Now we obtain a relation between the similar solutions $S$ and $K$ up to $O\left(\epsilon \epsilon_{m}\right)$ :

$$
\begin{aligned}
0= & \epsilon\{K(\tau)-2 S(\tau)\}-\epsilon^{2} S^{2}(\tau)+\epsilon \epsilon_{m}\left(\tau_{m}^{\chi \rightarrow \sigma}\left\langle\sigma_{m}^{\mathrm{BO}}\right\rangle \frac{d K}{d \tau}\right. \\
& \left.-\frac{d S}{d \tau}\right)+O\left(\epsilon\left(\epsilon_{m} \tau_{m}^{\chi \rightarrow \sigma}\left\langle\sigma_{m}^{\mathrm{BO}}\right\rangle\right)^{2}\right) .
\end{aligned}
$$

Taking account of the fact that $\epsilon_{m} \ll \epsilon \ll 1$ and $\epsilon \epsilon_{m} \ll \epsilon^{2} \ll \epsilon$ for sufficiently large $m$, the balance of the first and second terms on the right hand side of Eq. (5.7) up to $O\left(\epsilon^{2}\right)$ leads to

$$
K(\tau)=2 S(\tau)+\epsilon S(\tau)^{2} \simeq 2 S(\tau)
$$

The magnitudes of the other terms are equal to or less than $O\left(\epsilon \epsilon_{m} \tau_{m}^{\chi \rightarrow \sigma}\left\langle\sigma_{m}^{\mathrm{BO}}\right\rangle\right)$. We require that relations of different orders are separately satisfied in relation (5.7). In particular, the relation of the order $\epsilon \epsilon_{m}$ leads to

$$
\left\langle\sigma_{m}^{\mathrm{BO}}\right\rangle \tau_{m}^{\chi \rightarrow \sigma}=\frac{1}{2}
$$

This relation indicates that the induction time satisfies the BO time scaling $\tau_{m}^{\chi \rightarrow \sigma}=\left\langle\sigma_{m}^{\mathrm{BO}}\right\rangle^{-1} / 2 \sim \lambda^{-2 m / 5}$.

This relation is also proved by directly solving Eq. (4.1). Substituting $\sigma_{m}=\left\langle\sigma_{m}^{\mathrm{BO}}\right\rangle+\delta \sigma_{m}$ and $\chi_{m}=\left\langle\chi_{m}^{\mathrm{BO}}\right\rangle+\delta \chi_{m}$ into Eq. (4.1), we obtain the linearized equation with respect to $\delta \chi_{m}$ and $\delta \sigma_{m}$ by neglecting $\left(\delta \sigma_{m}\right)^{2}$ :

$$
\frac{d}{d t}\left(\delta \sigma_{m}\right)=(\alpha g) \delta \chi_{m}-2\left\langle\sigma_{m}^{\mathrm{BO}}\right\rangle \delta \sigma_{m}
$$

Then the solution is

$$
\delta \sigma_{m}(t)=\alpha g \int^{t} e^{-2\left\langle\sigma_{m}^{\mathrm{BO}}\right\rangle\left(t-t^{\prime}\right)} \delta \chi_{m}\left(t^{\prime}\right) d t^{\prime}
$$

Thus the characteristic time for $\delta \sigma_{m}$ is $\left\langle\sigma_{m}^{\mathrm{BO}}\right\rangle^{-1}$. If we regard this characteristic time as the induction time $\tau_{m}^{\chi \rightarrow \sigma}$, then it is shown that the induction time obeys the BO time scaling.

We can verify that the results obtained by numerical simulations satisfy these conclusions. Figures 11(b) and 11(d) show that condition (5.8) is satisfied: the peak of $K_{m}$ is twice as large as that of $S_{m}$. Figure 10 shows that the BO time scaling holds for $\tau_{m}^{\chi \rightarrow \sigma}$. It is also shown that $\left\langle\sigma_{m}^{\mathrm{BO}}\right\rangle \tau_{m}^{\chi \rightarrow \sigma}$ is almost constant and about 0.5 , which assures that the relation of order $\epsilon \epsilon_{m}$ on the right hand side of Eq. (5.7) holds.

In the same way as the derivation of the relation (5.7), we obtain the following equation from Eq. (4.2) for the inertial range:

$$
\epsilon \epsilon_{m} \frac{d K}{d \tau}=\epsilon \epsilon_{m} \lambda^{4 / 5}\left\langle\sigma_{m}^{\mathrm{BO}}\right\rangle\left(\frac{1}{2} \tau_{m-1}^{\sigma}+\tau_{m-1}^{\chi}+\tau_{m}^{\chi}\right) \frac{d K}{d \tau} .
$$

In this case, the balance relations of order $\epsilon$ and $\epsilon^{2}$ hold identically. Thus the condition for the existence of the similar solution $S$ is $\left\langle\sigma_{m}^{\mathrm{BO}}\right\rangle\left(\frac{1}{2} \tau_{m-1}^{\sigma}+\tau_{m-1}^{\chi}+\tau_{m}^{\chi}\right)=\lambda^{-4 / 5}$. In fact, 

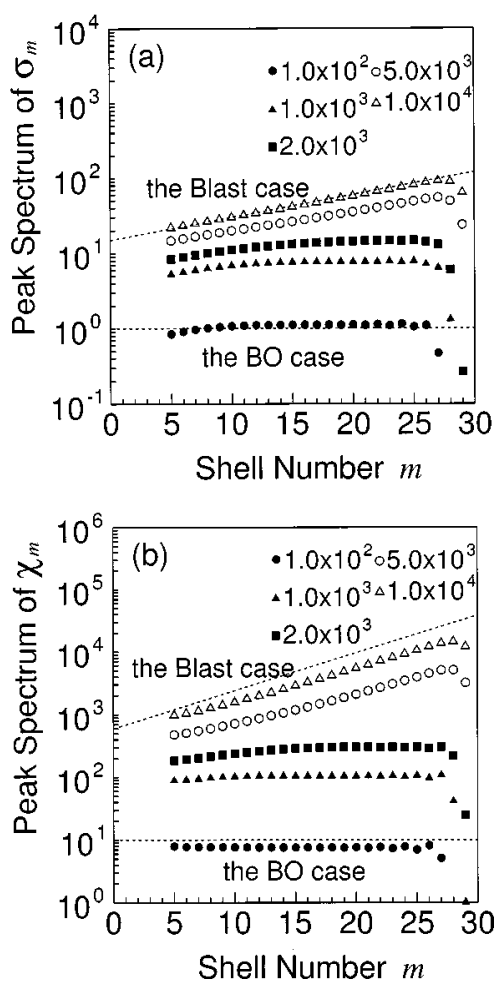

FIG. 15. Peak spectra for different magnitudes of initial disturbance: $\delta \chi_{4}=10^{2}, 10^{3}, 2 \times 10^{3}, 5 \times 10^{3}$, and $10^{4}$. (a) Peak spectra for $\lambda^{2 / 5 m} \sigma_{m}$. (b) Peak spectra for $\lambda^{4 / 5 m} \chi_{m}$.

the numerically evaluated value of $\lambda^{4 / 5}\left\langle\sigma_{m}^{\mathrm{BO}}\right\rangle\left(\frac{1}{2} \tau_{m-1}^{\sigma}\right.$ $\left.+\tau_{m-1}^{\chi}+\tau_{m}^{\chi}\right)$ is independent of $m$ and about 1.0. This condition is classified into the following three conditions: (a) $\tau_{m}^{\chi} \sim\left\langle\sigma_{m}^{\mathrm{BO}}\right\rangle^{-1}$ and $\tau_{m}^{\sigma} \sim\left\langle\sigma_{m}^{\mathrm{BO}}\right\rangle^{-1}$, (b) $\tau_{m}^{\chi} \sim\left\langle\sigma_{m}^{\mathrm{BO}}\right\rangle^{-1}$ and $\tau_{m}^{\sigma}$ $\ll \tau_{m}^{\chi}$, and (c) $\tau_{m}^{\sigma} \sim\left\langle\sigma_{m}^{\mathrm{BO}}\right\rangle^{-1}$ and $\tau_{m}^{\chi} \ll \tau_{m}^{\sigma}$. Figure 10 shows that $\tau_{m}^{\sigma}$ is smaller than $\tau_{m}^{\chi}$ and the ratio of $\frac{1}{2} \tau_{m-1}^{\sigma}$ to $\tau_{m}^{\chi}$ is about 0.2 , which also means that $\tau_{m}^{\sigma}$ is not so important for the existence of the similar solutions.

Relation (5.8), $K(\tau)=2 S(\tau)$, can be confirmed by Figs. 7 and 8 for DNS's and by Fig. 11 for our shell model. For DNS's, three pairs of the fluctuations $A_{X 4}$ and $A_{\Sigma 4}, B_{X 5}$ and $B_{\Sigma 5}$, and $B_{X 6}$ and $B_{\Sigma 6}$ are chosen to confirm relation (5.8). High correlations are due to the similarity of the functions $X_{m}(t)$ and $\Sigma_{m}(t)$. Moreover, the shape of the pair $A_{X 4}$ and $A_{\Sigma 4}$ is different from that of the pairs $B_{X 5}$ and $B_{\Sigma 5}$ and $B_{X 6}$, and $B_{\Sigma 6}$. Thus this is consistent with the result of the perturbation analysis that $S_{m}$ and $K_{m}$ must be almost similar, but their shapes are not determined uniquely. The similar shapes of $S_{m}$ and $K_{m}$ can be dependent differently on initial conditions. In fact, other similar solutions $S_{m}^{\prime}$ and $K_{m}^{\prime}$ derived from another initial condition are shown in Fig. 16. The similar solutions $S^{\prime}$ and $K^{\prime}$ are different in shape from those in Fig. 11. Therefore, we conclude that our shell model can well explain the results of DNS's.

In this analysis, we assume $|\tau| \ll 1$ or $t \approx 0$. Although a little bit severe, this condition can be interpreted as follows. The period $\tau \sim-1$ corresponds to the initial stage, i.e., $t$ $\sim 0$. In this stage, the modes excited dominantly are not in the inertial range, as shown in Fig. 11. Since these modes indirectly affect the inertial range, the modes $S_{m}$ or $K_{m}$ belonging to the inertial range do not necessarily satisfy the
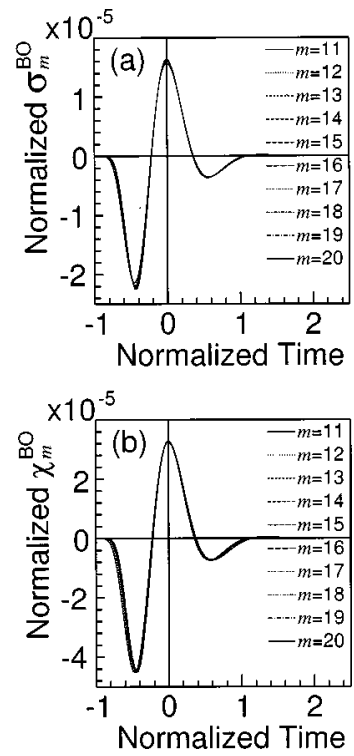

FIG. 16. $S_{m}\left(\tau_{m}\right)$ and $T_{m}\left(\tau_{m}^{\prime}\right)$ for another initial disturbance $\delta \chi_{m=5}=-1.0 \times 10^{-2}$. (a) $S_{m}\left(\tau_{m}\right)$ in the inertial range $(11<m$ $<20)$. (b) $T_{m}\left(\tau_{m}^{\prime}\right)$ in the inertial range $(11<m<20)$.

similarity for the initial stage. On the other hand, the period $\tau>0$ corresponds to $t>t_{\infty}$ for sufficiently large $m$ when an infinite number of modes has been excited without dissipative effect. This period is not realistic, because we could not continue simulation after $t_{\infty}$. In real simulations, solutions exist even after $t_{\infty}$ because of dissipation.

The condition $|\tau| \ll 1$ can be relaxed as $|\tau|<1$, because the similarity of $S$ and $K$ holds even for $|\tau|<1$, as seen in Fig. 15. This suggests that even in an initial stage, the effect of transient variations of large scales on the modes in the inertial range becomes weak as the considered scale goes deeply into the inertial range. It is quite interesting that the similarity is sustained for $t>t_{\infty}$, though we cannot explain why the similarity holds even after the dissipation becomes effective. We have not been able to explain the similarity observed in the blast case.

\section{CONCLUDING REMARKS}

In this paper, we investigated the possibility of the existence of elementary processes constituting entropy cascade in free convection turbulence. We believe that entropy cascade is essentially similar to energy cascade: the cascades possess common essentials.

In the energy cascade picture, an eddy, i.e., a blob of energy, is successively broken into smaller ones within a so called turnover time. Although the picture is based on the statistics and scaling, the process described is quite dynamical. Thus it is suggested that in fully developed turbulence, energy is transferred to small scales by a number of elementary transfer processes, each of which corresponds to a sequence of breakdowns of a mother eddy into daughters for generations. During these processes, many generations correlate to each other even under the restriction of the localness of interactions. In fact, we observed characteristic entropy transfer processes in which correlating highly strong fluctuations of the entropy or $T$ vorticity $X_{m}$ are transferred through the inertial range. Thus these characteristic transfer processes 
seemed to be candidates for the elementary process of cascade. Each of these processes may correspond to the multigeneration picture of cascade introduced in Sec. I, because it has two different characteristic times: transfer time and staying time. The former obeys the BO time scaling. On the other hand, the latter is almost constant independent of scale although not so clearly defined in DNS's. The long-range correlation through many scales observed in characteristic transfer processes is a little bit curious, because in the traditional cascade picture there is only one characteristic time, and then it is believed that the correlation between scales decreases rapidly against the difference between them. However, by demanding only that the staying time is scaled the same way as the transfer time, the correlation among eddies (or $T$ vortices) of different scales does not decrease. Some randomness should be required for each of the breakdown processes of eddies. Thus the characteristic time seems to make sense only statistically in the traditional cascade picture.

We can observe two separable regions in a snapshot of an entropy field (see Fig. 1): a coherent part and a relatively random part. The latter probably corresponds to the traditional cascade picture. In this paper, to examine characteristic times we have introduced two quantities integrated over a shell, i.e., $X_{m}$ and $\Sigma_{m}$. Since the random part may be smoothed out in this integration, the substantial variations of $X_{m}$ and $\Sigma_{m}$ represent a temporal evolution of the coherent part. It should be noted that the variation itself is relatively small compared with the temporal average which obeys the BO scaling, and is contributed mainly by the random part. We infer that the characteristic transfer process mentioned above is related to the coherent part, i.e., coherent structures observed in a snapshot. Therefore, we conclude that the characteristic transfer process is not an elementary process of cascade but a manifestation of intermittency.

It is not natural that the transfer time of the characteristic transfer process obeys the $\mathrm{BO}$ time scaling, because each of the characteristic transfer processes is a dynamical process. The key point of this question is the existence of the random part, i.e., the background of the coherent part which can be approximated by the temporal averages $\left\langle X_{m}\right\rangle$ and $\left\langle\Sigma_{m}\right\rangle$. The scaling of this transfer time originates from the scaling of the background field.

We have confirmed this fact in terms of the shell model. It has been shown that small fluctuations of a large scale added to the steady solution which includes the BO scaling in its inertial range are transferred, satisfying the similarity. This similarity, proved by perturbation analysis, requires naturally that the transfer time should obey the BO time scaling. The success of the perturbation analysis indicates that the characteristic transfer process is a kind of linear process around the BO scaling. In this sense, the characteristic transfer process is never an elementary process of cascade.

Our shell model is based on the entropy cascade picture where the $T$ vorticity of a scale is stretched into smaller ones by a strain of the same scale induced by the buoyancy. In this picture, the dynamical evolution of some coherent structures of the $T$ vorticity is described, and any randomness is not included explicitly. If the traditional cascade only sustains the background fluctuation obeying the $\mathrm{BO}$ scaling statistically, our shell model includes only the average produced by the traditional cascade processes as the steady solution. This suggests that the dynamics of coherent structures is affected by the background. Although we do not clearly understand the characteristics of coherent structures and the relation to intermittency, this work suggests that the temporal evolution of coherent structures is a manifestation of intermittency.

We are interested in the meaning of the turnover time from a dynamical perspective. We have succeeded in defining the plausible characteristic time in terms of the correlation time. This characteristic time, however, is not the turnover time mentioned in the traditional cascade picture. This suggests that the traditional cascade is constituted not by successive but by random breakdown of eddies. In this sense, the turnover time should be regarded as not the transfer time but the staying time.

\section{APPENDIX A: 2D ORTHONORMAL WAVELET}

A one-dimensional orthonormal wavelet base, which is labeled by discretized parameters $j$ and $m$ corresponding to position and scale, is constructed by discrete scale transformation and translation as follows:

$$
\psi_{j}^{m}(x)=2^{m / 2} \psi_{0}\left(2^{m} x-j\right)(m, j \in \mathbb{Z}),
$$

where $\psi_{0}$ is a special function called the analyzing wavelet. The set $\left\{\psi_{j}^{m} \mid m, j \in \mathbb{Z}\right\}$ spans a one-dimensional complete orthonormal system. These wavelet bases are localized in both physical space and Fourier space, although the localness of them depends on their scales. The rough scale (wavelength) and position of a wavelet base characterized by parameters $(m, j)$ are $\sim 2^{-m}$ and $\sim 2^{-m} j$, respectively. We adopt Meyer's wavelet, which is infinitely differentiable, and its Fourier transform has compact support in Fourier space. The construction of Meyer's wavelet was introduced in Ref. [23].

To construct wavelet basis in two dimensions, we need another scaling function, the low-pass filter $\phi_{0}(x)$, in addition to the analyzing wavelet. The Fourier spectrum of $\phi_{0}(x)$ is located around $k=0$. Using these two scaling functions, a $2 \mathrm{D}$ wavelet base is composed of the three components

$$
\begin{aligned}
& \Psi_{m, j}^{(1)}(x, y)=\psi_{j 1}^{m}(x) \phi_{j 2}^{m}(y), \\
& \Psi_{m, j}^{(2)}(x, y)=\phi_{j 1}^{m}(x) \psi_{j 2}^{m}(y), \\
& \Psi_{m, j}^{(3)}(x, y)=\phi_{j 1}^{m}(x) \phi_{j 2}^{m}(y),
\end{aligned}
$$

where $\boldsymbol{j}=(j 1, j 2)$ indicates the position of a wavelet base in the form $\sim 2^{-m} \boldsymbol{j}$ and $\phi_{j}^{m}(x)$ is defined the same as $\psi_{j}^{m}(x)$, with $\psi_{0}(x)$ and not $\phi_{0}(x)$. In the $2 \mathrm{D}$ case the set $\left\{\Psi_{m, j}^{(q)} \mid j\right.$ $=(j 1, j 2) ; m, j 1, j 2 \in \mathbb{Z} ; q \in\{1,2,3\}\}$ is a complete orthonormal system. Any scalar field $f(x, y)$ is decomposed as

$$
f(x, y)=\sum_{m, j} \sum_{q=1}^{3} \hat{f}_{m, j}^{(q)} \Psi_{m, j}^{(q)}(x, y),
$$

where

$$
\hat{f}_{m, j}^{(q)} \equiv \int \Psi_{m, j}^{(q)}(x, y) f(x, y) d V
$$


For a 2D vector field, we decompose its $x$ and $y$ components separately.

\section{APPENDIX B: APPLICATION OF THE PERTURBATION ANALYSIS TO OTHER SHELL MODELS}

We can formally apply the perturbation analysis introduced in Sec. V to the original shell model for FC turbulence and a class of shell models for 3D NS turbulence. It should be noted that these shell models are chaotic systems unlike our model. Thus the following results merely indicate that these models possess the same similarity as ours.

The original shell model for FC turbulence $[18,19]$ is

$$
\begin{gathered}
\frac{d T_{m}}{d t}=A_{1} k_{m}\left(u_{m-1} T_{m-1}-\lambda u_{m} T_{m+1}\right)+A_{2} k_{m}\left(u_{m} T_{m-1}\right. \\
\left.-\lambda u_{m+1} T_{m+1}\right)-\kappa k_{m}^{2} T_{m}+f \delta_{m, 4}, \\
\frac{d u_{m}}{d t}=B_{1} k_{m}\left(u_{m-1}^{2}-\lambda u_{m} u_{m+1}\right)+B_{2} k_{m}\left(u_{m} u_{m-1}-\lambda u_{m+1}^{2}\right) \\
-\nu k_{m}^{2} u_{m}+\alpha g T_{m}-\left(\delta_{m, 1}+\delta_{m, 2}+\delta_{m, 3}+\delta_{m, 4}\right) \frac{1}{k_{m}^{2}} \bar{f},
\end{gathered}
$$

where $u_{m}$ and $T_{m}$ are representations of the velocity and temperature. The steady solution of Eqs. (B1) and (B2) in the inviscid case, $\bar{T}_{m}$ and $\bar{u}_{m}$, are

$$
\begin{aligned}
& \bar{T}_{m}=C k_{m}^{-1 / 5}, \\
& \bar{u}_{m}=D k_{m}^{-3 / 5},
\end{aligned}
$$

where $C$ and $D$ are constants which satisfy the following relation:

$$
0=D^{2} \lambda^{-1 / 5}\left(\lambda^{4 / 5}-1\right)\left(B_{1} \lambda^{3 / 5}+B_{2} \lambda^{-1 / 5}\right)+\alpha g C .
$$

We define nondimensional similar functions of velocity and temperature $U$ and $W$ as follows:

$$
\begin{gathered}
\epsilon U(\eta) \equiv \frac{u_{m}(t)-\bar{u}_{m}}{\bar{u}_{m}}, \\
\epsilon W\left(\eta^{\prime}\right) \equiv \frac{T_{m}(t)-\bar{T}_{m}}{\bar{T}_{m}} .
\end{gathered}
$$

The small parameter $\epsilon$ is introduced formally under the assumption that the order of the ratio of fluctuations to the steady solution is small. Thus $U$ and $W$ are of order 1 . The normalized times $\eta$ and $\eta^{\prime}$ are also defined on a particular shell $m$ as $\eta \equiv \tau_{m}^{u}=\left(t-t_{m}^{u}\right) / t_{m}^{u}$ and $\eta^{\prime} \equiv \tau_{m}^{T}=\left(t-t_{m}^{T}\right) / t_{m}^{T}$, where $t_{m}^{u}$ and $t_{m}^{T}$ are the times at which the right hand sides of Eqs. (B6) and (B7) peak, respectively.

Then similar solutions $U$ and $W$ exist if the following conditions hold:

$$
2 U(\eta)-W(\eta)=0
$$

$$
\begin{gathered}
B_{1}\left(2 \lambda^{4 / 5} \tau_{m-1}^{u}+\tau_{m}^{u}\right)+B_{2}\left(\lambda^{4 / 5} \tau_{m-1}^{u}+2 \tau_{m}^{u}\right)+\lambda^{-1 / 5}\left(\lambda^{4 / 5}-1\right) \\
\times\left(B_{1} \lambda^{3 / 5}+B_{2}\right) \tau_{m}^{T \rightarrow u} \sim k_{m}^{-2 / 5}, \\
\left(A_{1} \lambda^{4 / 5} \tau_{m-1}^{u}+A_{2} \lambda^{1 / 5} \tau_{m}^{u}\right)+\left(A_{1} \lambda^{4 / 5}+A_{2} \lambda^{1 / 5}\right)\left(\tau_{m-1}^{T}+\tau_{m}^{T}\right) \\
\sim k_{m}^{-2 / 5} .
\end{gathered}
$$

The condition (B8) is the same as relation (3.4) for our model. Conditions (B9) and (B10) mean some linear combinations of $\tau_{m}^{u}, \tau_{m}^{T}$, and $\tau_{m}^{T \rightarrow u}$ should be scaled as $\sim k_{m}^{-2 / 5}$. Thus characteristic times should satisfy the BO scaling even in this model.

A class of shell models for 3D NS turbulence also possesses the same property. Here we use the following shell model proposed by Ohkitani and Yamada [24]:

$$
\begin{aligned}
\left(\frac{d}{d t}+\nu k_{m}^{2}\right) u_{m}= & i k_{m}\left(a u_{m+1} u_{m+2}+b \lambda u_{m+1} u_{m-1}\right. \\
& \left.+c \lambda u_{m-1} u_{m-2}\right) *+f \delta_{m, 4} \\
& a+b \lambda+c \lambda^{2}=0
\end{aligned}
$$

where $a, b$, and $c$ are parameters which determine the properties of conserved quantities, one of which is the kinetic energy. The steady solution of Eqs. (B11) and (B12) in the inviscid case is

$$
\bar{u}_{m}=F k_{m}^{-1 / 3} \quad(\mathrm{~F}=\text { const })
$$

We define the nondimensional similar solution $U$ as follows:

$$
\epsilon U(\mu) \equiv \frac{u_{m}(t)-\bar{u}_{m}}{\bar{u}_{m}} .
$$

The small parameter $\epsilon$ is also introduced formally under the assumption that the order of the ratio of fluctuations to the steady solution is small. Thus $U$ is of order 1 . The normalized time $\mu$ is defined on a particular shell $m$ as $\mu \equiv \tau_{m}$ $=\left(t-t_{m}\right) / t_{m}$ where $t_{m}$ is the time at which the right hand side of Eq. (B14) peaks, respectively. Then we obtain the following leading order equation for $U$ :

$$
\frac{d U}{d \mu}=i F^{2 *} k_{m}^{2 / 3}\left(-a \lambda^{-1} \bar{\tau}_{m}+c \lambda^{2} \bar{\tau}_{m}\right) \frac{d U^{*}}{d \mu},
$$

where $\bar{\tau}_{m} \equiv \frac{1}{3}\left(\tau_{m-1}+\tau_{m}+\tau_{m+1}\right)$ is independent of shell number $m$ if

$$
-a \lambda^{-1} \bar{\tau}_{m}+c \lambda \bar{\tau}_{m-1} \sim k_{m}^{-2 / 3}
$$

Thus the characteristic time scale averaged with adjacent three terms must satisfy Kolmogorov's time scaling

$$
\bar{\tau}_{m} \sim k_{m}^{-2 / 3}
$$

to exist similar solution $U$. Though $U$ is complex, there is another constraint that nonzero $U$ exist. That is,

$$
\left|\bar{u}_{m}\right|^{2}\left(c \lambda \bar{\tau}_{m-1}-a \lambda^{-1} \bar{\tau}_{m}\right)>1,
$$

which is derived from Eq. (B15). 
[1] L. F. Richardson, Weather Prediction by Numerical Process (Cambridge University Press, Cambridge, 1922).

[2] Z.-S. She and E. Lévêque, Phys. Rev. Lett. 72, 336 (1994).

[3] A. N. Kolmogorov, Dokl. Akad. Nauk SSSR 31, 538 (1941).

[4] F. Heslot, B. Castaing, and A. Libchaber, Phys. Rev. A 36, 5870 (1987).

[5] B. Castaing, G. Gunaratne, F. Heslot, L. P. Kadanoff, and A. Libchaber, J. Fluid Mech. 204, 1 (1989).

[6] E. S. C. Ching, L. P. Kadanoff, A. Libchaber, and X.-Z. Wu, Physica D 58, 414 (1992).

[7] E. D. Siggia, Annu. Rev. Fluid Mech. 26, 137 (1994).

[8] T. Bolgiano, J. Geophys. Res. 64, 2226 (1959).

[9] A. M. Obukhov, Dokl. Akad. Nauk. SSSR 125, 1246 (1959).

[10] V. S. L'vov, Phys. Rev. Lett. 67, 687 (1991).

[11] S. Toh and E. Suzuki, Unstable and Turbulent Motion of Fluid (World Scientific, Singapore, 1993), p. 272.

[12] S. Toh and E. Suzuki, Phys. Rev. Lett. 73, 1501 (1994).
[13] S. Toh, J. Phys. Soc. Jpn. 64, 685 (1995).

[14] S. Kida and K. Ohkitani, Phys. Fluids A 4, 1602 (1992).

[15] A. Pumir and E. D. Siggia, Phys. Rev. Lett. 68, 1511 (1992).

[16] E. Weiman and C. W. Shu, Phys. Fluids 6, 49 (1994).

[17] E. Suzuki and S. Toh, RIMS Koukyuuroku 892, 195 (1995) (in Japanese).

[18] A. Brandenburg, Phys. Rev. Lett. 69, 605 (1992).

[19] E. Suzuki and S. Toh, Phys. Rev. E 51, 5628 (1995).

[20] M. Iima and S. Toh, RIMS Koukyuuroku 974, 170 (1996) (in Japanese).

[21] M. Iima and S. Toh, J. Phys. Soc. Jpn. 67, 4068 (1988).

[22] M. Iima and S. Toh, J. Phys. Soc. Jpn. 67, 373 (1998).

[23] Y. Meyer, in Wavelets, edited by J. M. Combes, A. Grossmann, and Ph. Tchamitchian (Springer, Berlin, 1988), p. 21.

[24] K. Ohkitani and M. Yamada, J. Phys. Soc. Jpn. 56, 4210 (1987). 